\title{
(Über)Leben nach Lungenversagen: Die DACAPO-Studie
}

Dodoo-Schittko F et al. German-wide prospective DACAPO cohort of survivors of the acute respiratory distress syndrome (ARDS): a cohort profile. BJM Open 2018; doi:10.1136/bmjopen2017-019342

Wie steht es um die Lebensqualität von Patienten nach akutem Lungenversagen? Mit welchen Spätfolgen haben sie zu kämpfen? Wie steht es um ihre Rückkehr ins Berufsleben? Dodoo-Schittko und Team haben dazu nun eine große Kohortenstudie begonnen, bei der sie Verlauf und intensivmedizinische Versorgungsqualität der Überlebenden als potenzielle Einflussfaktoren untersuchen und Betroffene in regelmäßigen Abständen befragen.

Etwa $10 \%$ aller ICU-Patienten entwickeln im Verlauf ein akutes Atemnotsyndrom (ARDS), in schweren Fällen liegt die Sterblichkeit sogar bei über $40 \%$. Zudem haben auch Überlebende meist schwer an einer überstandenden schweren Schädigung des Lungenparenchyms zu tragen: Jahre später leiden sie an körperlichen und mentalen Einschränkungen, die sich langfristig negativ auf ihre gesundheitsbezogene Lebensqualität auswirken können. Genau an diesem Punkt wollten Dodoo-Schittko und seine Kollegen ansetzen und haben zwischen September 2014 und Mai 2016 eine groß angelegte prospektive Kohortenstudie mit ARDS-Überlebenden begonnen. Dabei sollen vor allem die Auswirkungen der intensivmedizinischen Versorgungsqualität auf die Lebensqualität und die Rückkehr ins Berufsleben untersucht werden. Als Voraussetzung für die Studienteilnahme definierten die Untersucher zunächst folgende Kriterien:

- Diagnose eines ARDS auf Basis der aktuellen Berlin Kriterien,

- Alter über 18 Jahren zum Zeitpunkt der ARDS-Diagnose.

Zur Objektivierung der Versorgungsqualität auf ICU sammelten Dodoo-Schittko und Team sämtliche Details zum Verlauf inklusive soziodemografischer Parameter. Nach Entlassung sollen die Überlebenden zunächst 3 Monate und 6 Monate nach Entlassung und ab dann alle
12 Monate mittels Fragebogen zur Selbstbeurteilung zur gesundheitsbezogenen Lebensqualität und zur beruflichen Situation befragt werden. Die Studie ist daher bis heute noch nicht abgeschlossen, in der aktuellen Publikation geht es zunächst um das Gesamtprojekt und die ersten Ergebnisse.

Im Rekrutierungszeitraum erfüllten 1900 Patienten die Einschlusskriterien, 1225 gaben ihre Einwilligung und nahmen zunächst teil. Da leider 349 von ihnen verstarben, umfasst die aktuelle Kohorte an ARDS-Überlebenden 876 Patienten. 278 $(31,7 \%)$ sind weiblich, das durchschnittliche Lebensalter liegt bei 56,0 Jahren (44,0-66,0). 102 (11,8\%) Teilnehmer hatten ein leichtes ARDS, bei 406 $(47,1 \%)$ lag eine moderate und in 354 $(41,1 \%)$ Fällen eine schwere Verlaufsform vor. Die Ursachen konnten überwiegend als pulmonal beschrieben werden $(81,3 \%)$, der mittlere SOFA-Score zum Zeitpunkt der ARDS-Diagnose lag bei $8,0(6,0-11,0)$. Die genannten demografischen Ergebnisse decken sich laut Autoren mit entsprechenden Zahlen früherer Kohortenstudien und auch die hohe Prävalenz von Lungenerkrankungen ist mit der aktuellen Fachliteratur im Einklang. Als erste Ergebnisse in Hinblick auf den intensivmedizinischen Verlauf nennen die Forscher eine hohe Anzahl kritischer Ereignisse wie Hypoglykämien, den Einsatz von ECMO sowie Reintubationen.

FAZIT

Die vorgestellte Studie untersucht erstmals gezielt den Einfluss der intensivmedizinischen Behandlung auf die Lebensqualität und die berufliche Widereingliederung von ARDS-Überlebenden. Erste Ergebnisse decken sich mit den Zahlen bekannter Kohortenstudien zum Thema. Die ersten Resultate umfassen eine hohe Prävalenz pulmonaler Ursachen sowie hohe Komplikationsraten inklusive Reintubationen und ECMO.

Dipl.-Psych. Annika Simon, Hannover

\section{Kommentar}

Was wird eigentlich aus unseren Patienten nach Entlassung aus dem Krankenhaus? Zurecht beschäftigt diese Frage inzwischen viele Wissenschaftler! Dabei ist die Antwort angesichts der hohen Inzidenz von Depressionen, Angststörungen und anderen gesundheitlichen Einschränkungen ehemaliger Intensivpatienten leider ernüchternd.

Weitgehend unbekannt sind bislang die Einflussfaktoren und damit möglichen Stellschrauben während der Behandlung auf der Intensivstation. Mit der DACAPO-Studie haben Herr Prof. Thomas Bein und seine Kollegen nun eine einzigartige Multicenterstudie zur Untersuchung dieser Faktoren bei ARDS-Patienten in Deutschland realisiert. Die Datenanalysen versprechen Aufschluss sowohl hinsichtlich potenziell beeinflussbarer Faktoren wie dem Blutglukosespiegel oder dem Sedierungs- und Transfusionsregime, aber auch nicht veränderbaren, soziokulturellen Gegebenheiten wie dem Ausbildungsstatus, dem Familienstand oder den Einkommensverhältnissen. Die Anzahl der analysierten Datensätze ist mit 876 sehr hoch. Der geringe Anteil von Patienten mit leichtem ARDS spiegelt dabei wahrscheinlich die klinische Realität wider, in der das leichte ARDS häufig nicht als solches identifiziert wird, jedoch dürfen insbesondere für das moderate und schwere ARDS in den nächsten Monaten viele spannende Ergebnisse erwartet werden, wofür den Initiatoren der Studie und hier insbesondere Thomas Bein selbst schon heute gratuliert werden muss!

\section{Autorinnen/Autoren}

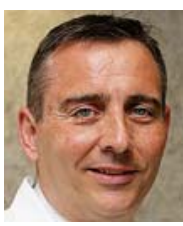

Prof. Dr. med. Rolf Dembinski, Klinik für Intensivmedizin und Notfallmedizin, Klinikum Bremen Mitte 